\title{
PREVALENCE OF GASTRO-INTESTINAL PARASITES IN CAPTIVE BIRDS AT KAMLA NEHRU ZOOLOGICAL GARDEN, KANKARIA ZOO, AHMEDABAD, GUJARAT
}

\author{
H.R. Parsani ${ }^{1}$, R.R. Momin ${ }^{2}$, R.K. Sahu ${ }^{3}$ and B.G. Patel ${ }^{4}$ \\ ${ }^{I}$ Veterinary Officer, ${ }^{2}$ I/C Professor and Head, Department of Parasitology, College of Veterinary Science and Animal Husbandry, Gujarat \\ Agricultural University, Sardar Krushinagar, Gujarat 385506, India. \\ ${ }^{3}$ Zoo Superintendent, ${ }^{4}$ Assistant Zoo Superintendent, Kankaria Zoo, Ahmedabad, Gujarat, India.
}

\begin{abstract}
A total of 138 group faecal samples collected cagewise of different wild birds from Kamla Nehru Zoological Garden, Kankaria Zoo, Ahmedabad were examined. Out of this $62(44.93 \%)$ were found positive for parasitic infection. Eggs of Ascaris and Capillaria species were observed in 25 (71.43\%) and $13(37.14 \%)$ samples respectively, while the oocyst of coccidia (Eimeria sp.) were observed in $53(85.48 \%)$ samples. Ova of trematode $8(12.90 \%)$ and cestode segment $3(4.84 \%)$ were also observed.
\end{abstract}

\section{Keywords}

Parasitic infection, zoo birds, Ascaris, Capillaria, Eimeria, Kamala Nehru Zoological Garden, Gujarat

\section{Introduction}

There are 27 natural orders and 8600 families representing 30,000 species of bird in the world. Out of which, in India to day there are 20 orders and 75 families representing 1200 species of birds (Ali, 1996). Inspite of providing all possible care and facilities, the birds kept in the zoo are under constant stress due to captivity and are prone to infections. Parasitic infections have caused considerable losses to wild life in this country. A number of parasites are responsible for illness and death of captive birds and exercise great influence on the success of bird management in the zoo. Since a systematic study of parasitic infections has not been conducted in Kamla Nehru Zoological Garden, Kankaria Zoo, Ahmedabad that houses 138 species of birds belonging to 24 families and totalling 2068 individuals, a study was undertaken and is communicated in this article.

\section{Materials and Methods}

Fresh group faecal samples of birds were collected cage wise in clean sterile containers from Kamla Nehru Zoological Garden, Kankaria Zoo Ahmedabad. A part of the sample was fixed in 10 per cent formalin and brought to the laboratory for direct concentration and centrifugation in saturated salt solution. While the other part of each sample was mixed with $2.5 \%$ potassium dichromate solution and kept at room temperature for sporulation of coccidian oocyst. Gross helminth pass in the faeces were identified after staining with borax carmine in case of trematodes and cestodes. Nematodes were studied after clearing them in lactophenol as per standard procedure.

\section{Results and Discussion}

The faecal examination of 13814 samples revealed 44.93 per cent parasitic infection with the highest prevalence of nematode eggs $(56.45 \%)$ and coccidian oocysts $(85.48 \%)$. The infection of trematodes was $12.90 \%$ in carnivorous birds while cestode infection was $4.84 \%$ in columbid birds ( Table 1). Earlier workers have reported parasitic infection varying from 25 to $99 \%$ in zoo birds - Baranga Zoo, Orissa (Patnaik \& Acharjyo, 1970); Lucknow Zoo, Uttar Pradesh (Pande et al., 1970); Lucknow and Delhi Zoo (Chauhan, et al., 1973); Mysore Zoo (Muraleedharan et al., 1990); Bannerghatta National Park, Bangalore (Reddy et al., 1992), and Ahmedabad and Baroda Zoo, Gujarat (Patel et al., 1998).

Species-wise nematode infection consisted of Ascaridia species (71.43\%), Capillaria species (37.14\%) and Strongyloid species (8.57\%). Patnaik and Acharjyo (1970), Chauhan et al., (1973), Islam (1991), Reddy et al., (1992), and Patel et al., (1998) reported nematode infection mainly with Ascaridia species and Capillaria species in galliformes and columbiformes birds. In this study also nematode infection mainly with Ascaridia species (71.43\%) and Capillaria species (37.14\%) in columbiformes and

Part of MVSc. thesis submitted to Gujarat Agricultural University, Sardar Krushinagar by first author

Received 12 November 2001; $\quad$ Revised received 18 October 2002; $\quad$ Finally accepted 30 November 2002 
gallifromes birds was recorded. It seems that nematode infection was $56.45 \%$ among all parasitic infections, which is high due to their direct life cycle.

Patnaik and Acharjyo (1970), Islam (1991), and Reddy et al., (1992) reported that cestode infection is mostly found in galliformes and columbiformes. In the present investigation $4.84 \%$ cestode infection was observed only in columbiformes birds as reported by Patel et al., (1998). Patnaik and Acharjyo (1970) reported trematode infection in ciconiformes and falconiformes birds. During the present study $12.90 \%$ trematode infection was observed in ciconiformes and falconiformes birds. Both trematode and cestode infections were found in low numbers which might be due to their indirect life cycle. Trematode infection in ciconiformes and falconiformes may be due to fish which may act as intermediate host.

\section{Conclusion}

In the present study nematode and coccidian infection were most commonly observed which might be due to their direct life cycle; whereas the trematode and cestode infection were low due their indirect life cycle. Trematode infection was mainly due to fish given to ciconiformes and falconiformes birds. Infected birds did not show any alarming clinical signs because of regular deworming at three months interval for three days in drinking water. However, some birds were not taking drug due to change of water taste. This might be a reason for parasites especially those that have direct life cycle to remain in the cage and reinfect the birds.

\section{Acknowledgements}

The authors are highly grateful to Dr. M.C. Desai, Principal and Dr. D.K. Pethkar, Former Professor of Parasitology, College of Veterinary Science and Animal Husbandry, Gujarat Agricultural University, Sardar Krushinagar for providing laboratory facilities and encouragement.

\section{References}

Ali, S. (1996). The Book of Indian Birds, $12^{\text {th }}$ edition. Oxford University Press, Oxford, NewYork, 187pp.

Chauhan P.P.S., B.B. Bhatia, G.S. Arora, R.D. Agarwal and S.S. Ahluwalia (1973). A preliminary survey of parasitic infection among mammals and birds at Lucknow and Delhi Zoo. Indian Journal of Animal Science 43: 163-168.

Islam, A.M.W. (1991). Incidence of helminth parasites of guinea fowl in Zambia. Abstract of International Seminar on Veterinary Medicine in Wild and Captive Animals Banglore p.28 (unpublished).

Reddy, J.N.R., M.S. Jagannath, P.E. D'souza and A.S. Rahman (1992). Prevalence of gastrointestinal parasites in wild mammals and captive birds at Bannerghatta National Park, Banglore, India. Indian Journal of Animal Science 62: 1046-1048.

Muraleedharan, K., V. Iswariah, S.K. Ziauddin and K. Srinivasan (1990). A survey of gastro intestinal parasites of mammals of Zoological Garden at Mysore. Mysore Journal of Agriculture Science 24: 250-
256.

Pande, B.P., B.B. Bhatia, P.P.S. Chauhan and R.K. Garg (1970). Species composition of coccidia of some of the mammals and birds at the zoological garden, Lucknow. Indian Journal of Animal Science 40: 154-163.

Patel P.V., A.I. Patel, R.K. Sahu, and R. Vyas (1998). Helminthic infection in captive wild birds of Gujarat. Abstract of the $10^{\text {th }}$ National Congress of Veterinary Parasitology, Jabalpur, 72pp. (unpublished).

Patnaik M.M. and L.N. Acharjyo (1970). Notes on the helminth parasites of vertebrates in Baranga Zoo (Orissa). Indian Veterinary Journal 47: 723-730. 
Table 1. Prevalence of parasitic infection in captive birds of Kamla Nehru Zoological Garden, Kankaria Zoo, Ahmedabad

\begin{tabular}{|c|c|c|c|c|c|c|}
\hline \multirow[t]{2}{*}{ English name } & \multirow[t]{2}{*}{ Scientific name } & \multirow{2}{*}{$\begin{array}{l}\text { No. of birds } \\
\text { infected }\end{array}$} & \multicolumn{4}{|c|}{ Type of infection } \\
\hline & & & Cestode & Nematode & Trematode & Eimerian oocyst \\
\hline \multicolumn{7}{|l|}{ Galliformes: Phasianidae } \\
\hline Indian Peafowl & Pavo cristatus & 11 & - & Capillaria sp. & - & - \\
\hline \multirow[t]{2}{*}{ Grey Peacock-pheasant } & Polyplectron bicalcaratum & 1 & - & Capillaria sp. & & \\
\hline & & & & Ascaridia sp. & - & - \\
\hline White Pea fowl & Pavo cristatus & 30 & - & Ascaridia sp. & - & 1 \\
\hline Bhutan Grey Peacock pheasant & Polypectron thibetanus & 4 & - & Capillaria sp. & - & 1 \\
\hline Edward Pheasant & Lophura edwardsi & 1 & - & - & - & - \\
\hline Golden Pheasant & Chrysolophus pictus & 3 & - & - & - & 1 \\
\hline Lady Amherst's pheasant & Chrysolophus amhersitiae & 4 & - & - & - & - \\
\hline Silver Pheasant & Lophura nycthemera & 17 & - & Capillaria sp. & - & 1 \\
\hline Red Junglefowl & Gallus gallus & 1 & - & - & - & - \\
\hline Grey Junglefowl & Gallus sonneratii & 2 & - & Ascaridia sp. & - & - \\
\hline Painted Spurfowl & Galloperdix lunulata & 1 & - & - & - & - \\
\hline Red Spurfowl & Galloperdix spadicea & 2 & - & - & - & 1 \\
\hline Jungle Bush-Quail & Perdiula asiatica & 4 & - & - & - & 1 \\
\hline Japanese Green Pheasant & Phasianus vericolor & 2 & - & - & - & - \\
\hline Palwan Peacock Pheasant & Polyplectron emphanum & 1 & - & - & - & - \\
\hline Grey Francolin & Francolinus pondicerianus & 3 & - & - & - & - \\
\hline Black Francolin & Francolinus francolinus & 3 & - & - & - & - \\
\hline \multicolumn{7}{|l|}{ Psittaciformes: Psittacidae } \\
\hline Cardinal Red-crested Parakeet & Paroaria cucullata & 1 & - & - & - & - \\
\hline Cockatiel & Nymphicus hollandicus & 61 & - & - & - & 1 \\
\hline Grey Cockatiel & Colopsitta hollandicus & 4 & - & - & - & - \\
\hline Cockatoo & Kakatoasp. & 3 & - & - & - & - \\
\hline Little Corella & Kakatoa senguinea & 4 & - & - & - & - \\
\hline Rose crested Cockato & Kakatoa moluccensis & 4 & - & - & - & 1 \\
\hline Goffin cockatoo & Kakatoa senguinea goffini & 2 & - & - & - & - \\
\hline Greater White Crested Cockatoo & Kakatoa alba & 3 & - & - & - & - \\
\hline Black-headed Connure & Aratinga sp. & 1 & - & - & - & - \\
\hline Brown-eared Connure & Aratinga sp. & 19 & - & Ascaridia sp. & - & 1 \\
\hline Jandaya Connure & Aratinga jandaya & 3 & - & - & - & - \\
\hline Pantagonian Connure & Aratinga sp. & 8 & - & - & - & 1 \\
\hline Peruvian Connure & Aratinga sp. & 1 & - & - & - & - \\
\hline Red-headed Connure & Aratinga wagleri & 4 & - & - & - & - \\
\hline Black Lory & Chalcopsitta sp. & 1 & - & - & - & - \\
\hline Black-capped Lory & Chalcopsitta atra petra & 3 & - & - & - & - \\
\hline Black-winged Lory & Eos cyanogenia & 1 & - & - & - & - \\
\hline Chattering Lory & Domicella garrula garrula & 4 & - & - & - & 1 \\
\hline Red Mollucan Lorry & Eos bornea bornea & 1 & - & - & - & - \\
\hline Blue and Yellow Macaw & Ara ararauna & 1 & - & - & - & - \\
\hline Illiger's Macaw & Ara maracana & 2 & - & - & - & - \\
\hline Scarlet Macaw & Ara sp. & 1 & - & - & - & - \\
\hline Yellow-collared Macaw & Ara auricollis & 2 & - & - & - & - \\
\hline Military Macaw & Ara militaris & 1 & - & - & - & - \\
\hline Yellow-headed Amazon & Amazona ochrocephala tresmariae & 1 & - & - & - & - \\
\hline Grey Parrot & Psittacus erithacus & 1 & - & - & - & - \\
\hline Senegal Parrot & Poicephalus senegalus & 2 & - & - & - & - \\
\hline Red-sided Electus & Lorious roratus pectoralis & 1 & - & - & - & - \\
\hline Parakeet & Psittacula sp. & 27 & - & - & - & 1 \\
\hline Alexandrine Parakeet & Psittacula eupatria & 30 & - & Ascaridia sp. & - & 1 \\
\hline Plum-headed Parakeet & Psittacula cyanocephala & 15 & - & - & - & - \\
\hline Rose-breasted Parakeet & Psittacula sp. & 22 & - & - & - & 1 \\
\hline Rose-ringed Parakeet & Psittacula krameri & 25 & - & - & - & 1 \\
\hline
\end{tabular}




\begin{tabular}{|c|c|c|c|c|c|c|}
\hline \multirow[t]{2}{*}{ English name } & \multirow[t]{2}{*}{ Scientific name } & \multirow{2}{*}{$\begin{array}{l}\text { No. of birds } \\
\text { infected }\end{array}$} & \multicolumn{4}{|c|}{ Type of infection } \\
\hline & & & Cestode & Nematode & Trematode & Eimerian oocyst \\
\hline Crimson-winged Parakeet & Aprosmictus erythropterus & 10 & - & - & - & 1 \\
\hline Crimson Rosella & Platycerus sp. & 2 & - & - & - & - \\
\hline Eastern Rosella & Platycerus eximinus & 2 & - & - & - & - \\
\hline \multicolumn{7}{|l|}{ Gruiformes: Rallidae } \\
\hline Common Moorhen & Gallinula chloropus & 2 & - & - & - & - \\
\hline Purple Moorhen & Porphyrio porphyrio & 1 & - & - & - & - \\
\hline White-breasted Moorhen & Amaurornis phoenicurus & 1 & - & - & - & - \\
\hline \multicolumn{7}{|l|}{ Gruidae } \\
\hline Common Crane & Grus grus & 1 & - & - & - & - \\
\hline Demoiselle Crane & Grus virgo & 7 & - & - & Lypersomasp. & 1 \\
\hline \multicolumn{7}{|l|}{ Anseriformes: Anatidae } \\
\hline Brahmini Shelduck & Tadorna ferruginea & 5 & - & Ascaridia sp. & - & 1 \\
\hline Comb Duck & Sarkidiornis melanatos & 10 & - & - & - & 1 \\
\hline Common Duck & Anas sp. & 27 & - & Ascaridia sp. & - & 1 \\
\hline Northen Pintail & Anas acuta & 2 & - & - & - & - \\
\hline Spot-billed Duck & Anas poecilorhyncha & 6 & - & - & - & - \\
\hline Japanese Duck & Anas sp. & 4 & - & - & - & - \\
\hline Mandarin Duck & Aix galericulata & 8 & - & - & - & 1 \\
\hline Common Teal & Anas crecca & 1 & - & - & - & - \\
\hline Lesser Whistling-Duck & Dendrocygnajavanica & 2 & - & - & - & - \\
\hline Black Swan & Chenopsis atratus & 5 & - & - & - & - \\
\hline Goose & Anser sp. & 6 & - & Ascaridia sp. & Lypersomasp. & - \\
\hline Bar-headed Goose & Anser indicus & 5 & - & - & Lypersoma sp. & 1 \\
\hline Canadian Goose & Branta canadensis & 1 & - & - & - & - \\
\hline Greylag Goose & Anser anser & 5 & - & - & - & 1 \\
\hline \multicolumn{7}{|c|}{ Ciconiiformes: Threskiornithidae } \\
\hline Eurasian Spoonbill & Platalea leucorodia & 8 & - & - & - & - \\
\hline Black Ibis & Pseudibis papillosa & 1 & - & - & - & - \\
\hline White Ibis & Threskiornis acthiopica & 24 & - & - & - & 1 \\
\hline \multicolumn{7}{|l|}{ Phalacrocoracidae } \\
\hline Little Cormorant & Phalacrocorax niger & 13 & - & - & - & 1 \\
\hline \multicolumn{7}{|l|}{ Ardeidae } \\
\hline Cattle Egret & Bubulcus ibis & 19 & - & Ascaridia sp. & - & 1 \\
\hline Grey Heron & Ardea cinerea & 1 & - & - & - & - \\
\hline Western Reef-egret & Egretta gularis & 6 & - & - & - & - \\
\hline Black-crowned Night-heron & Nycticorax nycticorax & 40 & - & - & Lypersomasp. & 1 \\
\hline Indian Pond-heron & Ardeola grayii & 1 & - & - & - & - \\
\hline \multicolumn{7}{|l|}{ Charadriidae } \\
\hline Red-wattled Lapwing & Vanellus indicus & 1 & - & - & - & - \\
\hline \multicolumn{7}{|l|}{ Phoenicopteridae } \\
\hline Flamingos & Phoenicopterus sp. & 31 & - & Ascaridia sp. & - & 1 \\
\hline \multicolumn{7}{|l|}{ Pelecanidae } \\
\hline Dalmatian Pelican & Pelecanus crispus & 1 & - & - & - & - \\
\hline Great White Pelican & Pelecanus onocrotalus & 17 & - & - & Lypersomasp. & 1 \\
\hline
\end{tabular}




\begin{tabular}{|c|c|c|c|c|c|c|}
\hline \multirow[t]{2}{*}{ English name } & \multirow[t]{2}{*}{ Scientific name } & \multirow{2}{*}{$\begin{array}{l}\text { No. of birds } \\
\text { infected }\end{array}$} & \multicolumn{4}{|c|}{ Type of infection } \\
\hline & & & Cestode & Nematode & Trematode & Eimerian oocyst \\
\hline \multicolumn{7}{|l|}{ Laridae } \\
\hline Brown-headed Gull & Larus brannicephalus & 3 & - & - & - & - \\
\hline \multicolumn{7}{|l|}{ Recurvirostridae } \\
\hline Black-winged Stint & Himantopus himantopus & 1 & - & - & - & - \\
\hline \multicolumn{7}{|l|}{ Ciconidae } \\
\hline Greater Adjutant-stork & Leptoptilos dubius & 1 & - & - & - & - \\
\hline Black Stork & Ciconia nigra & 1 & - & - & - & - \\
\hline Black-necked Stork & Ephippiorhynchus asiaticus & 2 & - & - & Lypersoma sp. & 1 \\
\hline Painted Stork & Mycteria leucocephala & 1 & - & - & - & 1 \\
\hline European White Stork & Ciconia ciconia & 2 & - & Capillaria sp. & Lypersoma sp. & - \\
\hline White-necked Stork & Ciconia episcopus & 1 & - & - & - & - \\
\hline \multicolumn{7}{|l|}{ Accipitridae } \\
\hline Black Vulture & Sarcogyps sp. & 1 & - & - & - & 1 \\
\hline Red-headed Vulture & Sarcogyps calvus & 3 & - & Ascaridia sp. & Lypersoma sp. & - \\
\hline Indian White-backed Vulture & Gyps bengalensis & 8 & - & Ascaridia sp. & - & 1 \\
\hline \multicolumn{7}{|l|}{ Casuariiformes: Casuaridae } \\
\hline Cassowary & Casuarius casuarius & 1 & - & - & - & - \\
\hline Emu & Dromaius novaehollandicus & 7 & - & Ascaridia sp. & - & 1 \\
\hline \multicolumn{7}{|l|}{ Bucerotiformes: Bucerotidae } \\
\hline Indian Grey Hornbill & Ocyceros birostoris & 3 & - & - & - & - \\
\hline \multicolumn{7}{|l|}{ Cucliformes: Cuclidae } \\
\hline White cheeked Turaco & Turaco leucotis & 1 & - & - & - & - \\
\hline \multicolumn{7}{|l|}{ Columbiformes: Columbidae } \\
\hline Nicobar Pigeon & Caloenas nicobarica & 19 & - & Strongyloid sp. & - & 1 \\
\hline \multirow[t]{2}{*}{ Crowned Pigeon } & Goura cristata & 9 & - & Strongyloid sp. & & \\
\hline & & & & Ascaridia sp. & - & - \\
\hline Green Imperial-Pigeon & Ducula aenea & 2 & - & - & - & - \\
\hline Pied Imperial-Pigeon & Ducula bicolor & 2 & - & - & - & - \\
\hline Victoria Crowned Pigeon & Goura victoria & 2 & - & - & - & - \\
\hline Dove & - & 6 & - & Capillaria sp. & - & - \\
\hline Oriental Turtle-Dove & Streptopelia orientalis & 2 & - & - & - & 1 \\
\hline Diamond Dove & Geopelia cuneata & 4 & - & Capillaria sp. & - & 1 \\
\hline Jacobin Pigeon & Columba sp. & 23 & & $\begin{array}{l}\text { Capillaria sp. } \\
\text { Raillietina sp. }\end{array}$ & - & 1 \\
\hline \multirow[t]{2}{*}{ Green Fan-tail Pigeon } & Columba sp. & 50 & - & Ascaridia sp. & & \\
\hline & & & & Capillaria sp. & - & 1 \\
\hline \multirow[t]{2}{*}{ Black Fan-tail Pigeon } & Columba sp. & 56 & - & Capillaria sp. & & \\
\hline & & & & Strongyloid sp. & - & 1 \\
\hline \multirow[t]{3}{*}{ White Fan- tail Pigeon } & Columba sp. & 55 & & Ascaridia sp. & & \\
\hline & & & & Raillietina sp. & & \\
\hline & & & & Capillaria sp. & - & - \\
\hline Shiraji Pigeon & Columba sp. & 17 & - & Capillaria sp. & - & 1 \\
\hline Phouter Pigeon & Columba sp. & 28 & - & Ascaridia sp. & - & 1 \\
\hline \multirow[t]{2}{*}{ Khal Pigeon } & Columba sp. & 238 & & $\begin{array}{l}\text { Ascaridia sp. } \\
\text { Raillietina sp. }\end{array}$ & & \\
\hline & & & & Capillaria sp. & - & 1 \\
\hline Broze hybrid Pigeon & Columba sp. & 25 & - & Ascaridia sp. & - & 1 \\
\hline
\end{tabular}




\begin{tabular}{|c|c|c|c|c|c|c|}
\hline \multirow[t]{2}{*}{ English name } & \multirow[t]{2}{*}{ Scientific name } & \multirow[t]{2}{*}{ No. of birds } & \multicolumn{4}{|c|}{ Type of infection } \\
\hline & & & Cestode & Nematode & Trematode & Eimerian oocyst \\
\hline \multicolumn{7}{|l|}{ Passeriformes: Passeridae } \\
\hline Finches & Teaniopygia sp. & 43 & - & Ascaridia sp. & - & 1 \\
\hline Golden-cherry Love-bird & Agapornis sp. & 48 & - & - & - & 1 \\
\hline Blue Peach-faced Love-bird & Agapornis sp. & 134 & - & Ascaridia sp. & - & 1 \\
\hline Peach-faced Love-bird & Agapornis sp. & 98 & - & - & - & - \\
\hline Fischer's Love-bird & Agapornis fischerii & 150 & - & Ascaridia sp. & - & 1 \\
\hline \multicolumn{7}{|l|}{ Estrilidae } \\
\hline Red Munia & Amandava amandava & 4 & - & - & - & - \\
\hline Spotted Munia & Lonchura punctulata & 5 & - & - & - & 1 \\
\hline Three coloured Munia & - & 4 & - & - & - & - \\
\hline White-throated Munia & Lonchura malabarica & 5 & - & - & - & - \\
\hline Black-headed Munia & Lonchura malacca & 5 & - & - & - & - \\
\hline \multicolumn{7}{|l|}{ Passerinae } \\
\hline Common Sparrow & Passer domesticus & 1 & - & - & - & - \\
\hline Java Sparow & Padda oryzivora & 108 & - & Ascaridia sp. & - & 1 \\
\hline Budgeriger & - & 292 & - & Ascaridia sp. & - & 1 \\
\hline Canary & - & 5 & - & - & - & - \\
\hline \multicolumn{7}{|l|}{ Pycnonotidae } \\
\hline Red-vented Bulbul & Pycnonotus cafer & 2 & - & - & - & - \\
\hline \multicolumn{7}{|l|}{ Corvidae } \\
\hline White Crow & Corvus splendens & 2 & - & Ascaridia sp. & - & 1 \\
\hline Koel & Eudynamys scolopacea & 2 & - & - & - & - \\
\hline \multicolumn{7}{|l|}{ Turdinae } \\
\hline Pakin Robbin & - & 1 & - & - & - & - \\
\hline \multicolumn{7}{|l|}{ Sturnidae } \\
\hline Brahmini Starling & Sturnus pagodarum & 3 & - & - & - & - \\
\hline Common Myna & Acridotheres tristis & 2 & - & - & - & - \\
\hline Jungle Myna & Acridotheres fuscus & 5 & - & - & - & 1 \\
\hline Starling Myna & - & 1 & - & - & - & - \\
\hline
\end{tabular}

